\title{
La variabilidad de la frecuencia cardiaca en el control del entrenamiento en un corredor de Ironman. Estudio de caso Heart Rate Varibility in the training monitoring of an Ironman runner. A case study
}

*Claudio Nieto-Jimenez, **José Ruso-Álvarez, ***Elena Pardos-Mainer, **Jose Naranjo Orellana *Universidad del Desarrollo (Chile), **Universidad Pablo de Olavide (España), ***Universidad San Jorge (España)

\begin{abstract}
Resumen. El objetivo de este artículo es el de proporcionar información sobre la carga de entrenamiento a partir del seguimiento de un atleta durante siete meses de preparación para una competición de Ironman (3.8km de natación, 180 km de ciclismo y 42.195 km de carrera), utilizando una metodología de entrenamiento con registros diarios de variabilidad de la frecuencia cardiaca (VFC). Como variable para evaluar la actividad parasimpática se calculó la raíz cuadrada de la media de las diferencias de la suma de los cuadrados entre intervalos RR adyacentes (RMSSD) y el índice de estrés (SS) como indicador de actividad simpática. En los meses dos, tres y cuatro presentó un moderado incremento del SS $(d=1,17)$ paralelamente a una moderada disminución de la $\operatorname{RMSSD}(d=-1,04)$. Para los meses cinco, seis, y siete se observó una pequeña disminución del SS $(d=-0,23)$ y moderado aumento de la $\operatorname{RMSSD}(d=1,08)$. Como concusión es que las mediciones de VFC de cinco minutos al despertar parecen ser una forma útil de monitorizar el estado de equilibrio parasimpático en un triatleta antes de abordar las sesiones de entrenamiento. El análisis de esta monitorización serviría para detectar precozmente posibles estados de fatiga y para poder modificar, en caso necesario, la planificación de las cargas de entrenamiento en la preparación para una competición de Ironman.
\end{abstract}

Palabras clave: Variabilidad de la frecuencia Cardíaca, Ironman, Carga de entrenamiento, RMSSD.

Summary. The objective of this article is to provide information on the relationship between external (TL) and internal training load (ITL) from the tracking of an athlete during seven months of preparation for an Ironman competition (3.8 km of swimming, $180 \mathrm{~km}$ of cycling, and 42,195 km of running), using a training methodology with daily records of the heart rate variability (HRV). As a variable to assess parasympathetic activity, the square root of the mean of the differences in the sum of the squares between adjacent RR intervals (RMSSD) was calculated, together with the stress score (SS) as an indicator of sympathetic activity. In months two, three and four, there was a moderate increase in SS ( $d=1.17)$ parallel to a moderate decrease in RMSSD $(d=-1.04)$. In months five, six, and seven, a small decrease in SS $(d=-0.23)$ and a moderate increase in RMSSD $(d=1.08)$ were observed. As a conclusion, the five-minute HRV measurements upon awakening appear to be a useful tool to monitor the state of parasympathetic equilibrium in a triathlete before approaching training sessions. The analysis of this monitoring would serve to detect possible fatigue states in the early stages and to modify, if necessary, the training load planning in preparation for an Ironman competition.

Keywords: Heart Rate Variability, Ironman, Training load, RMSSD.

\section{Introducción}

En los últimos años, ha existido gran interés por controlar la carga de entrenamiento en los deportistas con el objetivo de monitorizar su adaptación, tanto positiva como negativamente (Bourdon, Cardinale, Murray, Gastin, Kellmann, Varley \& Cable, 2017). Distinguiéndose en la literatura, dos tipos de cargas de entrenamiento, por una parte, la carga administrada o carga de entrenamiento externa (CE), y por otra, la forma en la que cada sujeto asimila esa carga administrada y responde a ella, que es lo que se conoce como carga de entrenamiento interna (CI) (Halson, 2014, McLaren, Macpherson, Coutts, Hurst, Spears, \& Weston, 2018).

En este apartado, se ha observado el aporte de la variabilidad de la frecuencia cardiaca (VFC) como un método no invasivo para determinar la implicación del sistema nervioso autónomo (SNA) en estados de sobre-entrenamiento y fatiga (Buchheit, Racinais, Bilsborough, Bourdon, Voss, Hocking \& Coutts, 2013, Buchheit, 2014), así como asimilación de la CE (Sarabia Cachadiña, Cruz Torres, \& Naranjo Orellana, 2012, Nieto-Jiménez, Pardos-Mainer, Ruso-Álvarez, \& NaranjoOrellana, 2019). Una de las variables de VFC más utilizadas para explorar la CI es la raíz cuadrada de la media de las diferencias de la suma de los cuadrados entre intervalos RR adyacentes (RMSSD) (Task Force, 1996) siendo el estadísti-

Fecha recepción: 06-08-19. Fecha de aceptación: 05-10-19 claudio nieto-jimenez

c.nieto@udd.cl co parasimpático más utilizado en el deporte debido al menor coeficiente de variación en comparación con otros índices (Plews, Laursen, Stanley, Kilding, \& Buchheit, 2013, Le Meur, Pichon, Schaal, Schmitt, Louis, Gueneron \& Hausswirth, 2013, Michael, Jay, Halaki, Graham, \& Davis, 2016). Hay autores que utilizan directamente la RMSSD (o su Ln) para el seguimiento diario en deportistas (Thorpe, Strudwick, Buchheit, Atkinson, Drust, \& Gregson, 2015, Flatt, Esco, \& Nakamura, 2017, Ravé \& Fortrat 2016) mientras que otros proponen utilizar el coeficiente de variación (CV) de esta variable a lo largo de los días. (Flatt et al., 2017, Nakamura, Pereira, Rabelo, Flatt, Esco, Bertollo \& Loturco, 2016). En esta línea, el CV en deportistas profesionales ha presentado fluctuaciones entre el 4 y 9\% del LnRMSSD durante bloques de entrenamiento en remeros de élite (Plews et al., 2013) y triatletas de élite (Plews et al., 2012, Le Meur et al., 2013).

Además, el gráfico de dispersión de Poincaré proporciona información acerca de las ramas simpática y parasimpática del sistema nervioso autónomo (Tulppo, Makikallio, Takala, Seppanen, \& Huikuri, 1996) a través de sus diámetros transversal (SD1) y longitudinal (SD2). A partir de aquí, dado que el SD2 es un indicador inverso de actividad simpática, se puede calcular el índice de estrés (SS) como el inverso de SD2 multiplicado por 1000 como un indicador directo de actividad simpática. (Naranjo, De la Cruz, Sarabia, De Hoyo, \& Dominguez Cobo, 2015). Además, Naranjo y colaboradores (Naranjo, De la Cruz, Sarabia, De Hoyo, \& Dominguez, 2015) aportaron varias relaciones en las que se fortalece la idea de que, efectivamente, el SS es un indicador de actividad simpá- 
tica al igual que el SD2 (inverso). Esto abrió una perspectiva en el análisis de la VFC, ya que el SS y la relación simpáticoparasimpático (S: PS) permitirían ser una herramienta útil para monitorizar los cambios del balance autonómico a partir de variables convencionales de VFC.

El triatlón tiene distintas modalidades (Revelles, 2017), siendo el Ironman una competición que incluye $3.8 \mathrm{~km}$ de natación, 180 km de ciclismo y 42.195 km de carrera. Uno de los elementos a tomar en cuenta para controlar la CE es el equilibrio simpático-parasimpático, al ser un importante factor en atletas de resistencia, especialmente al estudiar la vuelta a la calma después del ejercicio, es decir, su proceso de recuperación para enfrentar una nueva jornada de entrenamiento.

El objetivo de este estudio de caso es proporcionar información sobre el seguimiento de un atleta durante siete meses de preparación para una competición de Ironman, utilizando registros diarios de VFC como medida de la CI.

\section{Materiales y Métodos}

\section{Participante}

Se monitorizó a un atleta masculino de categoría amateur nacional (edad 46 años; talla $175 \mathrm{~cm}$; peso $76.5 \mathrm{~kg}$ ) durante una temporada competitiva de siete meses que incluyó una competición de Ironman (3.8km de natación, 180 km de ciclismo y $42.195 \mathrm{~km}$ de carrera). Las sesiones de entrenamiento fueron planificadas de acuerdo con el objetivo, utilizándose como cuantificación de la carga realizada (CE) la distancia recorrida y el tiempo de entrenamiento acumulado. Fueron 195 días de registros, siendo la competición el día 179.

El atleta cumplimentó un cuestionario médico para descartar que estuviera recibiendo tratamientos o que sufriera trastornos cardiovasculares o de otro tipo que pudieran alterar el estado del sistema nervioso autónomo. El sujeto fue informado del procedimiento que se seguiría y dio su consentimiento por escrito para participar en el estudio, que siguió los principios expresados en la Declaración de Helsinki (The World Medical Association, 2018).

\section{Procedimiento. Registros de datos de VFC}

Durante 195 días consecutivos se realizaron mediciones basales de VFC a las 06:00 am en posición decúbito supino durante cinco minutos. Se utilizó un pulsómetro Polar V800 (Polar, Kempele, Finland) con banda torácica, validado para mediciones de VFC (Giles, Draper, \& Neil, 2015). La serie de tiempo RR de este dispositivo fue descargada mediante cable USB utilizando la correspondiente aplicación de la marca Polar FlowSync (versión 3.0.0.1337, Kempele, Finland) y posteriormente analizada con el software Kubios HRV (versión 3.1.0, University of Eastern Finland, Kuopio, Finland). Cada registro se inspeccionó previamente para detectar la posible presencia de artefactos y/o latidos anómalos, aplicando en caso necesario los filtros correspondientes ofrecidos por el propio software, sin rebasar en ningún caso el filtro medio.

Como variable del dominio de tiempo para evaluar la actividad parasimpática se calculó la RMSSD en ms (Task Force, 1996). Por otra parte, a partir del SD2 del gráfico de dispersión de Poincaré (Tulppo et al., 1996) se calculó el índice de estrés (SS) (Naranjo et al., 2015) como indicador de actividad simpática. Por último, el cociente entre el SS y SD1 nos informó de la relación entre simpático y parasimpático (Ratio S/Ps) (Naranjo Orellana et al., 2015).

\section{Análisis Estadístico}

Los datos se presentan como media y desviación estándar (DE) con su correspondiente coeficiente de variación (CV). Se midió el tamaño del efecto (TE) mediante el cálculo de la «d» de Cohen para un intervalo de confianza del 90\% y utilizando los rangos de interpretación propuestos por Hopkins (Hopkins, Marshall, Batterham, \& Hanin, 2009): trivial (menor de 0,2); pequeño (de 0,20 a 0,59); moderado (de 0,6 a 1,2) y grande (mayor de 1,2).

Para analizar correlaciones entre variables se utilizó el coeficiente de correlación de Pearson (r). Los análisis estadísticos se realizaron mediante el paquete estadístico SPSS ${ }^{\circledR}$, versión 22.0.

\section{Resultados}

En la tabla 1 Se muestran el promedio de carga Externa (CE) expresada en tiempo y kilómetros para cada uno de los mesociclos para la ciclismo, carrera y natación. Los resultados se expresan como media \pm desviación estándar.

En la tabla 2 se muestran el tiempo de entrenamiento y la RMSSD, SS y ratio S/Ps en los siete Mesociclos. Los resultados se expresan en medias, desviación estándar (DS), coeficiente de variación (CV) y Tamaño del Efecto (d).

En la figura 1 Se muestra la evolución del tiempo en horas y su relación con las semanas de entrenamiento

En la figura 2 Se muestra la correlación entre las medias semanales del tiempo de entrenamiento con la RMSSD (A) y SS (B). El coeficiente de correlación de Pearson fue de $\mathrm{r}=$ 0,06 con la RMSSD y $r=0,15$ con el SS.

En la figura 3 se presenta la evolución de los registros diarios matutinos de las variables de RMSSD y SS mostrando en líneas horizontales los rangos de normalidad para ambas variables. Se indica la competición Ironman realizada el día 179.

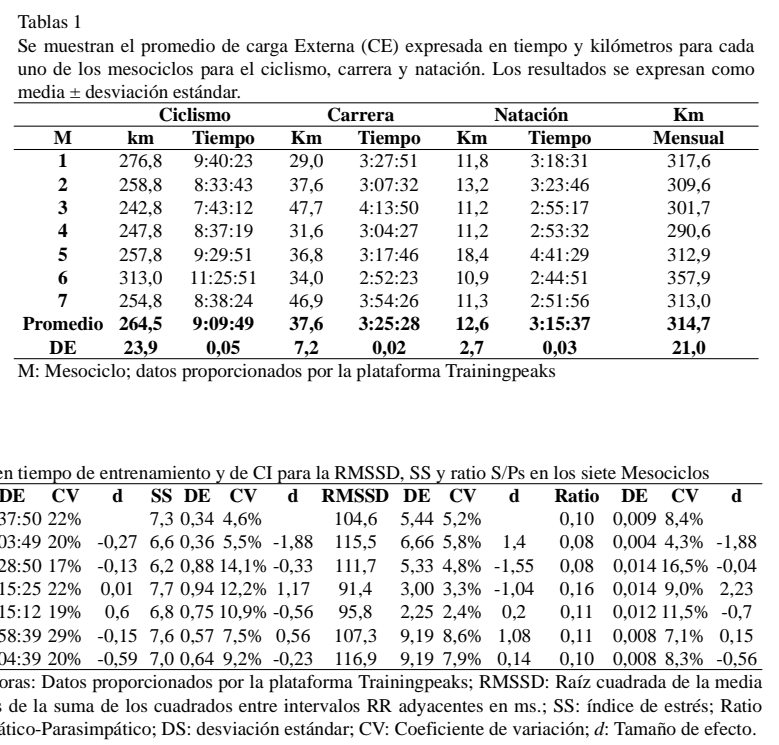




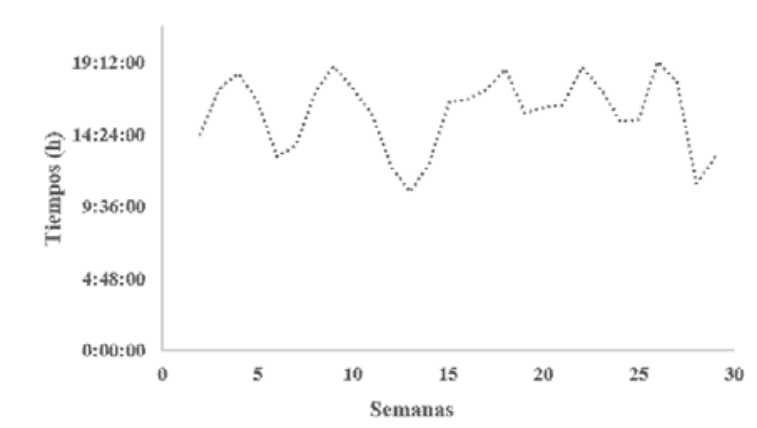

Figura 1. En la figura 1 se muestra la evolución del tiempo de entrenamiento en horas y su relación con las semanas de entrenamiento

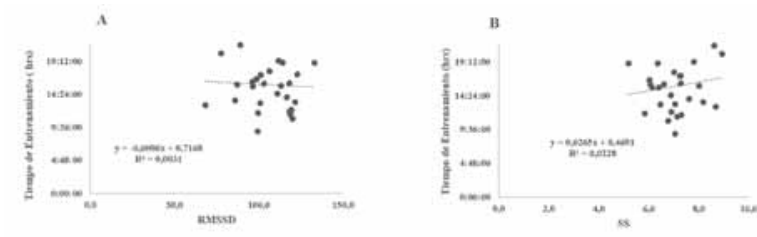

Figura 2. En la figura 2 se muestra la correlación entre las medias semanales del Tiempo de entrenamiento con la RMSSD (A) y SS (B). El coeficiente de correlación de Pearson fue de r = $-0,06$ con la RMSSD y $r=0,15$ con el SS.
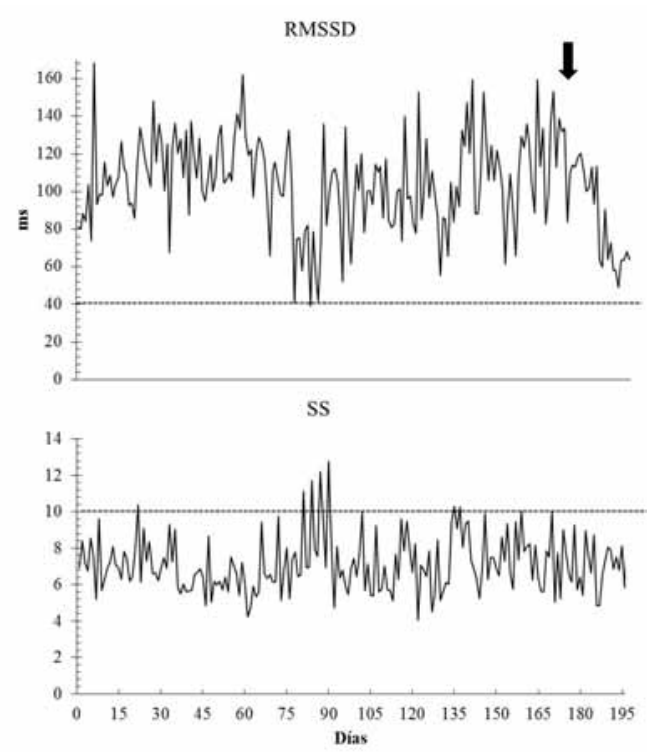

Figura 3. Evolución de los registros diarios matutinos de las variables de RMSSD y SS. RMSSD: Raíz cuadrada de la media de las diferencias de la suma de los cuadrados entre intervalos RR adyacentes en ms.; SS: Índice de estrés. Se indica la competición de Ironman el día 179. Las líneas segmentadas indican las zonas de alarma para el SS y la RMSSD.

\section{Discusión}

La principal contribución de este caso fue que los registros diarios matutinos de cinco minutos de duración de la RMSSD y el SS permitirían detectar cambios en la respuesta de un atleta al entrenamiento. La falta de correlación entre los volúmenes de entrenamiento y los datos de RMSSD y SS, es un dato a favor de que estas medidas de VFC sean un indicador de carga interna. Esto mismo se ha podido comprobar con anterioridad en triatletas femeninas (Nieto-Jiménez et al, 2019.).

El tiempo de entrenamiento durante los siete mesociclos de carga fue dividido en las tres disciplinas, donde el mayor tiempo fue para el ciclismo (tabla 1), esto concuerda con lo observado por Molina y colaboradores (Molina, Martínez, Lozano, Peinado, Montero, \& Molinuevo, 2010) que los triatletas entrenan distancias menores en cada una de las disciplinas comparados con los especialistas de cada depor- te, no obstante, el total de volumen de entrenamiento de cada sesión es mayor en los triatletas que en los especialistas (Hellemans, 1993; Sleamaker \& Browning, 1996, Molina et al, 2010). En nuestros datos, la media semanal del tiempo de entrenamiento para las tres disciplinas fue de 15 hrs 50 $\min 54 \operatorname{seg}$ (tabla 1$)$.

En nuestro estudio, la adaptación al entrenamiento dependía del control del volumen e intensidad como variables de CE y de los registros de VFC como variables de CI al despertar, para evitar el sobre-entrenamiento. La estrategia de medir basalmente cada mañana tenía el propósito de conocer en qué condiciones nuestro sujeto enfrentaba la nueva sesión de entrenamiento y monitorizar el diseño de las cargas.

La evolución del tiempo de entrenamiento (figura 1) en los meses dos, tres y cuatro presentó un moderado incremento del SS $(d=1,17)$ paralelamente a una moderada disminución de la RMSSD $(d=-1,04)$. Para los meses cinco, seis, y siete se observó una pequeña disminución del SS $(d=-0,23)$ y moderado aumento de la $\operatorname{RMSSD}(d=1,08)$ (Tabla 2). Con respecto al ratio $\mathrm{S} / \mathrm{Ps}$, se observó un gran aumento en los meses dos, tres y cuatro $(d=2,23)$ y una pequeña disminución para los meses cinco, seis, y siete $(d=-0,56)$. Por tanto, las CI que supusieron las diferentes sesiones no fueron anormalmente altas, al presentar TE pequeños y moderados a pesar de las variaciones observadas del tiempo de entrenamiento.

Dado que nuestro interés era observar una rutina de seguimiento diario, fue razonable pensar que el coeficiente de variación (CV) estaría aportando una información importante cuando se trata de valorar el estado del sistema simpáticoparasimpático en relación al volumen de entrenamiento (figura 1), al menos en este atleta en particular.

Algunos autores han recomendado las mediciones de VFC durante varios días a la semana para posteriormente realizar un análisis del promedio (Buchheit et al., 2013; Le Meur et al., 2013; Plews, Laursen, Stanley, Kilding, \& Buchheit, 2013) y otros aconsejan utilizar como herramienta de valoración el CV de los valores obtenidos (Flatt, Esco, \& Nakamura, 2017; Plews, Laursen, Kilding, \& Buchheit, 2012). En nuestro caso, el CV para para el SS fluctúo entre el 5 y el $14 \%$ y entre el 2 y el $8 \%$ para la RMSSD (Tabla 2). Además, observamos que durante los bloques de mayor carga (semana 5-6) el atleta presenta menores CV para el SS (entre 7 y $11 \%$ ) que en los bloques de menor carga (semana 3-4) (entre 12 y 14\%). Podríamos asumir que la falta de correlación entre los volúmenes de entrenamiento (figura 2) y los datos de SS $(r=0,15)$ y la RMSSD $(r=-0,06)$ es un dato a favor de que estas medidas de VFC sean un indicador de CI no existiendo ninguna relación con la CE. Este significado fisiológico es muy relevante, pues implica que existió una buena planificación de los periodos de trabajo y recuperación, permitiendo al organismo adaptarse desde el punto de vista biológico al estar sus valores de VFC en rangos normales.

En este sentido, existen tablas de percentiles para todas las variables de los diferentes dominios de la VFC (Corrales, de la Cruz, Garrido, Garrido \& Naranjo Orellana, 2012). En el caso del SS como medida de estrés simpático (Naranjo et al., 2015), sus autores recomiendan la utilización de los percentiles 75 al 90 como zona de alarma (Naranjo, De la Cruz, Sarabia, 
De Hoyo, \& Dominguez-Cobo, 2015) lo que equivale a un SS entre ocho y diez. Los valores medios semanales del SS que mostró este deportista (Tabla 2) se encuentran en general por debajo de 7.75 (percentil 75), a excepción de los días 84, 86,88 y 90 en que presentó valores mayores a 10. El valor del SS nos indicará si el tono simpático observado en situación basal se encuentra dentro de lo esperable en una recuperación completa o, por el contrario, muestra alguna alteración secundaria al impacto estresor ocasionado por la carga anterior situación que también ha sido observado en mujeres triatletas que han preparado una competición de Ironman (Nieto-Jiménez et al., 2019). Para la RMSSD los valores medios semanales que presentó el atleta (Tabla 2) se encuentran comprendidos entre los percentiles 75 (91 ms) y 95 (111 ms) para hombres deportistas (Corrales et al., 2012), de esta forma, su medición diaria en situación basal nos está indicando el grado de recuperación del tono parasimpático tras la carga realizada el día anterior. Por último, los valores de la Ratio S/PS (tabla 3) se encuentran muy por debajo del valor de 0,25 propuesto por Naranjo et al (2015), lo que reflejaría un balance autónomo totalmente equilibrado en los registros basales.

La RMSSD y el SS presentan una gran sensibilidad a los cambios durante el periodo de entrenamiento (figura 3), mostrando valores normales al despertar (por encima de $40 \mathrm{~ms}$ para la RMSSD y por debajo de 10 para el SS). Aun así, siguiendo la propuesta de Buchheit et al. (2013), Le Meur et al. (2013) y Plews et al. (2013), se realizaron los promedios semanales y mensuales. Parece ser que la respuesta parasimpática y simpática que presentó este atleta a las cargas administradas indicaría adaptaciones positivas al proceso de entrenamiento al estar sus valores en rangos normales sin señales de alarma para fatiga o sobreentrenamiento

En conclusión, las mediciones de VFC de cinco minutos al despertar parecen ser una forma útil de monitorizar el estado de equilibrio-parasimpático en un triatleta antes de abordar las sesiones de entrenamiento. El análisis de esta monitorización podría servir para detectar precozmente posibles estados de fatiga y para poder modificar, en caso necesario, la planificación de las cargas de entrenamiento. La selección de mediciones sencillas (RMSSD y el SS) permitió que esta metodología sea fácil de utilizar durante un periodo preparatorio para un Ironman, diferenciando la CI de la CE.

\section{Referencias}

Bourdon, P. C., Cardinale, M., Murray,A., Gastin, P., Kellmann, M., Varley, M. C., ... Cable, N. T. (2017). Monitoring Athlete Training Loads/ : Consensus Statement. International Journal of Physiology and Performance, 12(S2), 161170. https://doi.org/10.1123/IJSPP.2017-0208

Buchheit, M., Racinais, S., Bilsborough, J. C., Bourdon, P. C., Voss, S. C., Hocking, J., ... Coutts, A. J. (2013). Monitoring fitness, fatigue and running performance during a preseason training camp in elite football players. Journal of Science and Medicine in Sport, 16(6), 550-555. https:// doi.org/10.1016/j.jsams.2012.12.003.

Buchheit, M. (2014). Monitoring training status with HR measures: do all roads lead to Rome?. Frontiers in physiology, 5, 73.
Corrales, M. M., de la Cruz Torres, B., Garrido Esquivel, A., Garrido Salazar, M. A., \& Naranjo Orellana, J. (2012). Normal values of heart rate variability at rest in a young, healthy and active Mexican population. Health, 4(7), 377385. https://doi.org/10.4236/health.2012.47060

Revelles, A. B. F. (2017). Correlación en triatlón masculino entre fases y resultado final en los JJOO de Sídney 2000. Retos: nuevas tendencias en educación física, deporte y recreación, (32), 167-171.

Flatt, A. A., Esco, M. R., \& Nakamura, F. Y. (2017). Individual heart rate variability responses to preseason training in high level female soccer players. Journal of Strength and Conditioning Research, 31(2), 531-538. https:// doi.org/10.1519/JSC.0000000000001482

Flatt, A. A., Esco, M. R., Nakamura, F. Y., \& Plews, D. J. (2017). Interpreting daily heart rate variability changes in collegiate female soccer players. The Journal of Sports Medicine and Physical Fitness, 57(6), 907-915. https:// doi.org/10.23736/S0022-4707.16.06322-2

Giles, D., Draper, N., \& Neil, W. (2015). Validity of the Polar V800 heart rate monitor to measure RR intervals at rest. European Journal of Applied Physiology, 116(3), 563571. https://doi.org/10.1007/s00421-015-3303-9

Halson, S. L. (2014). Monitoring Training Load to Understand Fatigue in Athletes. Sports Medicine, 44, 139-147. https:/ /doi.org/10.1007/s40279-014-0253-z

Hellemans J. Triathlon: a complete guide for training and racing. Auckland: Reed; 1993

Hopkins, W. G., Marshall, S. W., Batterham, A. M., \& Hanin, J. (2009). Progressive statistics for studies in sports medicine and exercise science. Medicine and Science in Sports and Exercise, 41(1), 3-12. https://doi.org/10.1249/ MSS.0b013e31818cb278

Le Meur, Y., Pichon, A., Schaal, K., Schmitt, L., Louis, J., Gueneron, J., ... Hausswirth, C. (2013). Evidence of parasympathetic hyperactivity in functionally overreached athletes. Medicine and Science in Sports and Exercise, 45(11), 2061-2071. https://doi.org/10.1249/ MSS.0b013e3182980125

McLaren, S. J., Macpherson, T. W., Coutts, A. J., Hurst, C., Spears, I. R., \& Weston, M. (2018). The Relationships Between Internal and External Measures of Training Load and Intensity in Team Sports: A Meta-Analysis. Sports Medicine, 48(3), 641-658. https://doi.org/10.1007/s40279017-0830-z

Michael, S., Jay, O., Halaki, M., Graham, K., \& Davis, G. M. (2016). Submaximal exercise intensity modulates acute post-exercise heart rate variability. European Journal of Applied Physiology, 116(4), 697-706. https://doi.org/ 10.1007/s00421-016-3327-9

Molina, V. D., Martínez, E. D., Lozano, A. B. P., Peinado, P. J. B., Montero, F. J. C., \& Molinuevo, J. S. (2010). Control biológico del sobreentrenamiento en un mesociclo precompetitivo en triatletas de élite: un estudio piloto. Archivos de medicina del deporte: (135), 31-40.

Morton, R. H., Fitz-Clarke, J. R., \& Banister, E. W. (1990). Modeling human performance in running. Journal of Applied Physiology, 69(3), 1171-1177.

Nakamura, F. Y., Pereira, L. A., Rabelo, F. N., Flatt, A. A., Esco, M. R., Bertollo, M., \& Loturco, I. (2016). Monitoring 
weekly heart rate variability in futsal players during the preseason: the importance of maintaining high vagal activity. Journal of Sports Sciences, 34(24), 2262-2268. https://doi.org/10.1080/02640414.2016.1186282

Naranjo, J., De la Cruz, B., Sarabia, E., De Hoyo, M., \& Dominguez-Cobo, S. (2015). Heart Rate Variability/ : a Follow-up in Elite Soccer Players Throughout the Season. International Journal of Sports Medicine, 36, 881-886. https://doi.org/10.1055/s-0035-1550047

Naranjo Orellana, J., De la Cruz Torres, B., Sarabia Cachadiña, E., De Hoyo, M., \& Dominguez Cobo, S. (2015). Two New Indexes for the Assessment of Autonomic Balance in Elite Soccer Players. International Journal of Sports Physiology and Performance, 10, 452-457. https:// doi.org/http://dx.doi.org/10.1123/ijspp.2014-0235

Corrales, M. M., de la Cruz Torres, B., Esquivel, A. G., Salazar, M. A. G., \& Orellana, J. N. (2012). Normal values of heart rate variability at rest in a young, healthy and active Mexican population. Health, 4(7), 720-726.

Nieto-Jiménez, C., Pardos-Mainer, E., Ruso-Álvarez, J. F., \& Naranjo-Orellana, J. (In press). Training Load and HRV in a Female Athlete: A Case Study. Revista Internacional de Medicina y Ciencias de La Actividad Física y El Deporte. Recuperado de http://cdeporte.rediris.es/revista/inpress/artcarga1143e.pdf

Plews, D. J., Laursen, P. B., Kilding, A. E., \& Buchheit, M. (2012). Heart rate variability in elite triathletes, is variation in variability the key to effective training? A case comparison. European Journal of Applied Physiology, 112(11), 3729-3741. https://doi.org/10.1007/s00421-0122354-4

Plews, D. J., Laursen, P. B., Stanley, J., Kilding, A. E., \& Buchheit, M. (2013). Training adaptation and heart rate variability in elite endurance athletes: Opening the door to effective monitoring. Sports Medicine, 43(9), 773-781. https://doi.org/10.1007/s40279-013-0071-8

Pyne, D. B., \& Martin, D. T. (2011). Fatigue - insights from individual and team sports. In F. E. Marino (Ed.), Regulation of Fatigue in Exercise (pp. 177-186). New York: Nova Publishers.

Ravé, G., \& Fortrat, J. O. (2016). Heart rate variability in the standing position reflects training adaptation in professional soccer players. European Journal of Applied Physiology, 116(8), 1575-1582. https://doi.org/ 10.1007/s00421-016-3416-9

Sarabia Cachadiña, E., Cruz Torres, B., \& Naranjo Orellana, J. (2012). Estudio comparativo de los perfiles semanales de creatin kinasa, urea y variabilidad de la frecuencia cardiaca en remeros de élite españoles. Archivos de Medicina Del Deporte, XXIX(152), 952-958.

Sleamaker R, Browning R. Serious training for endurance athletes. 2nd ed. Champaign, IL.: Human Kinetics; 1996.

Task Force of The European Society of Cardiology and The North American Society of Pacing and Electrophysiology. (1996). Heart rate variability. Standards of measurement, physiological interpretation, and clinical use. European Heart Journal, 17, 354-381. https://doi.org/10.1161/ 01.CIR.93.5.1043

Thorpe, R. T., Strudwick, A. J., Buchheit, M., Atkinson, G., Drust, B., \& Gregson, W. (2015). Monitoring Fatigue During the In-Season Competitive Phase in Elite Soccer Players. International Journal of Sports and Performance, 10, 958-964. https://doi.org/10.1123/ijspp.2015-0004

Tulppo, M. P., Makikallio, T. H., Takala, T. E. S., Seppanen, T., \& Huikuri, H. V. (1996). Quantitative beat-to-beat analysis of heart rate dynamics during exercise. American Journal of Physiology, 271, H244-H252. https://doi.org/https:// doi.org/10.1152/ajpheart.1996.271.1.H244

WMA Declaration of Helsinki-Ethical Principles for Medical Research Involving Human Subjects - WMA - The World Medical Association. (2018). Recuperado de https:/ /www.wma.net/policies-post/wma-declaration-ofhelsinki-ethical-principles-for-medical-researchinvolving-human-subjects/
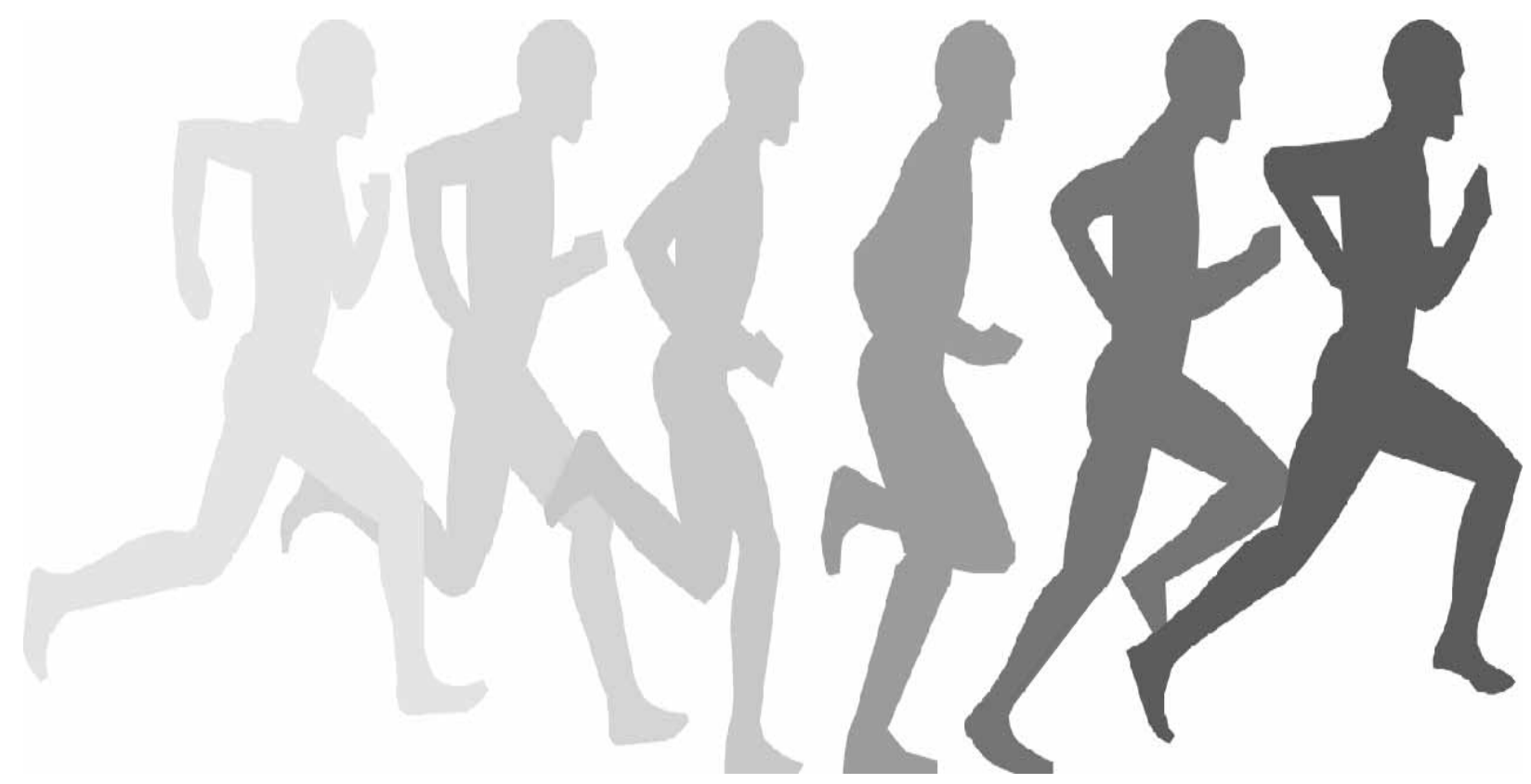